\title{
Evaluation Model of the Visual Fatigue on the 3D Stereoscopic Video
}

\author{
Gil-Ja So, Sang-Hyun Kim, and Jeong-Yeop Kim
}

\begin{abstract}
We propose content dependent factors to be an argument of evaluation model of visual fatigue. The content factors we choose are the strength and size of the excessive disparity range, the complexity of the background objects, the variation of the motion-depth, the contrast of the objects in the scene. We verify that these factors have a relationship with visual fatigue through the experiment and suggest methods to extract the degree of these factors automatically.
\end{abstract}

Index Terms-Content based fatigue factors, evaluation model of fatigue, stereoscopy, depth discontinuity, excessive disparity, fatigue of color.

\section{INTRODUCTION}

Each eye has its own viewpoint and receives slightly different images and receives depth perception cues psychologically and physiologically. Psychological cues include perspective, overlap, air perspective, shadow, apparent size, texture, etc. Physiological cues include binocular parallax, motion parallax, accommodation, and convergence [1].

To date, Stereoscopic image is made from depth that is constructed based on the difference between these two images, left and right image and it has been possible to realize true stereoscopic images. Conflict occurs between convergence or divergence eye movement, binocular disparity can be easily changed whereas the screen position or image position produced in the air by an optical systems cannot be changed easily, thus cause visual fatigue. Visual fatigue includes headaches, tiredness.

Thus, over the last decades, safety and health issues related to $3 \mathrm{D}$ stereoscopic video have been extensively studied.

The causes of visual fatigue are very diverse, and therefore, there needs ongoing research. There have been known that excessive binocular disparity, accommodation and convergence mismatch, DOF revisited, beyond the zone of comfortable viewing, within the zone of comfortable viewing, stereoscopic distortions, an artificial DOF, blur, etc. With

Manuscript received November 29, 2014; revised May 4, 2015. This research is partially supported by Ministry of Science, ICT and Future Planning (MSIP) and Institute for Information \&communications Technology Promotion (IITP) in the Information Communication Technology (ICT) and Culture Technology (CT) Research \&Development Program 2014.

Gil-Ja So is with the Department of Cyber Police and Science, University of Young-San, 288 Junam-ro, Yangsan-si Kyungsangnam-do 626-790, Korea (e-mail: kjso@ysu.ac.kr).

Sang-Hyun Kim is with the Department of Computer Engineering, University of Young-San, 288 Junam-ro, Yangsan-si Kyungsangnam-do 626-790, Korea (e-mail: ksh50@ysu.ac.kr).

Jeong-Yeop Kim is with University of Young-San, 99 Pilbong-gil Haeundae-Gu, Busan, 612-743, Korea (e-mail: neocopy@ysu.ac.kr). respect to the limit of disparity for comfortable viewing, the one degree of disparity appears to prevent the classical causes such as excessive binocular disparity and accommodation-convergence conflict, from being perceptually annoying [2]. Hence, visual fatigue cannot be evaluated with only one indicator.

There are many researches to evaluate the visual fatigue of stereoscopic video. [3] described the effects of the range of parallax distribution and temporal parallax changes. [4] showed the relationship between convergence eye movement and the accommodation function, and evaluate the visual fatigue both degree of parallax and amount of motion in stereoscopic image. [5], [6] determined the proper index of visual fatigue for quantitative analysis and evaluated the capability that viewers can recover the visual fatigue. [7] examine visual discomfort caused by motion-in-depth in terms of viewing time and display size in watching stereoscopic $3 \mathrm{D}$ video. They used a subjective test such as a questionnaire, and an objective test such as eye blink rate detection as assessment methods for visual fatigue.

However, previous studies did not consider content factors like hue information in evaluating visual fatigue. [8] introduced several content factors like as excessive binocular disparity, motion characteristics, binocular asymmetry, depth cue conflict, etc. Jung et al. [9] examined the relation between visual discomfort and object motion characteristics in stereoscopic 3D videos.

In this paper, we focus on some basic components in stereoscopic 3D content, the size and strength of the excessive disparity range, the complexity of the background object, the perceived depth of the hue, and the variation of the disparity between frames in pixels, as arguments of the evaluation model. Evaluation of visual fatigue is done using subjective methods mostly. 1SSQ, the simulator sickness questionnaire developed by Kenney et al. is a well known and a well established useful measurement tool for evaluating motion sickness caused by motion images [10]. Kuze and Ukai used a newly developed questionnaire to subjectively asses symptoms caused by viewing various types of motion images [11].

In this research, we propose five factors can be extracted from the stereoscopic content automatically for 3D evaluation model of visual fatigue. In experiment, we show that theses five factors induce visual fatigue and can be an argument of the 3D fatigue evaluation model using subjective method.

The rest of this paper is organized as follows : Section II reviews previous research for assessment of the visual fatigue or discomfort . Section III introduces five content factors to be able to induce visual fatigue and fatigue model with these factors, then describes the methods to predict the degree of 
content-based fatigue. Section IV shows that content-based factors proposed in this paper can be a reliable factor to predict visual fatigue and can be an argument of the fatigue evaluation metrics in experiment.

\section{PREVIOUS RESEARCH}

\section{A. An Assessment of Visual Discomfort Caused by Motion-in-Depth}

Cho \& Kang [7] provide assessment methods for visual discomfort through the subjective test and objective test. They used a questionnaire as a subjective test and eye blink rate detection as objective test. They used motion, binocular disparity, viewing time and display size as factors for assessment of visual discomfort.

Generally, motion-in-depth induces changes in binocular disparity, which derives the temporally changing demand of accommodation-vergence linkage. It was reported that the perceptual impact of excessive demand on the AC linkage is related to visual discomfort. So, they showed the relationship between visual discomfort and motion-in-depth with respect to diverse speed, viewing time and display size.

Visual discomfort was measured with a subjective assessment results based on the participants' questionnaires. As a result, 6 questions were statistically significant. Discomfort value is set as an average of these 6 questions rating scores.

Subjective visual discomfort was modeled using the polynomial function

$v(d, t)=\rho_{0}+\rho_{1} d+\rho_{2} t+\rho_{3} d^{2}+\rho_{4} d t+\rho_{5} t^{2}$, where $d$ denotes binocular disparity in degree $t$ is viewing time.

To convert eye blinking rates into objective visual discomfort, they correlate the eye blinking rate with viewer's visual discomfort responses. The relationship between eye blinking rates and visual discomfort is modeled using polynomial function

$h(b)=\phi_{0}+\phi_{1} b+\phi_{2} b^{2}$, where $b$ denotes the normalized eye blink rate.

By integrating eye blinking rates and viewer's discomfort responses, they construct an objective visual discomfort model. For each size of display, the observed eye blink rate corresponds to visual discomfort value by function $h$. From the calculate visual discomfort, they use the polynomial function

$u(d, t)=\gamma_{0}+\gamma_{1} d+\gamma_{2} t+\gamma_{3} d^{2}+\gamma_{4} d t+\gamma_{5} t^{2} \quad$, where $d$ denotes binocular disparity and $t$ is the viewing time to obtain the objective visual discomfort model.

\section{PROPOSED METHOD}

\section{A. Fatigue Model with Content Based Factors}

Despite of the perceptual significance of disparity magnitude, considering only global statics of disparity magnitude may not predict accurately subjective visual discomfort. Although two stereo images had the same disparity magnitude, human subjects tended to report different degrees of visual discomfort according to characteristics of scene elements [12]. However, the conventional visual comfort metrics have mostly focused on utilizing simple disparity magnitude statistics of stereoscopic 3D images. Thus, the conventional visual comfort metrics may not predict accurately subjective visual comfort.

In this paper, we propose content-dependent factors to predict visual fatigue and the model with these factors using polynomial function like as the model provided by Cho \& Kang. Meanwhile Cho \& Kang used motion-in-depth, content-based factor, viewing time and display size, environment-based factors, our model is just only composed of content-based factors.

We select 5 fatigue factor as arguments of the evaluation model, the size of excessive disparity range, the strength of excessive disparity range, the complexity of the background object, the variation of the disparity, the fatigue value of the color, contrast, of the content. The model to obtain visual fatigue value is defined using the polynomial function by (1).

$$
\begin{gathered}
F(t)=\alpha_{1} O_{\text {size }}(t)+\alpha_{2} O_{\text {strength }}(t)+\alpha_{3} C_{\text {contrast }}(t) \\
\alpha_{4} D_{\text {variation }}(t)+\alpha_{5} B_{\text {complexity }}(t)+C_{\text {cons } \tan t},
\end{gathered}
$$

where $F(t)$ is the polynomial function to obtain visual fatigue during duration time t, $O_{\text {size }}(t)$ is the size of excessive disparity range, $O_{\text {strength }}(t)$ is the strength of excessive disparity range, $C_{\text {contrast }}(t)$ is the fatigue value of the contrast, $B_{\text {complexity }}(t)$ is the complexity of the background object, $D_{\text {variation }}(t)$ is the variation of the disparity between frames.

As depicted in Fig. 1, the evaluation systems to extract the degree of the visual factor from 3D stereoscopic video is composed of the 4 sub systems for evaluation of perceived depth with contrast, extraction of the object with excessive disparities, extraction of the depth discontinuities and calculating the difference of disparity between pre and pro frames.

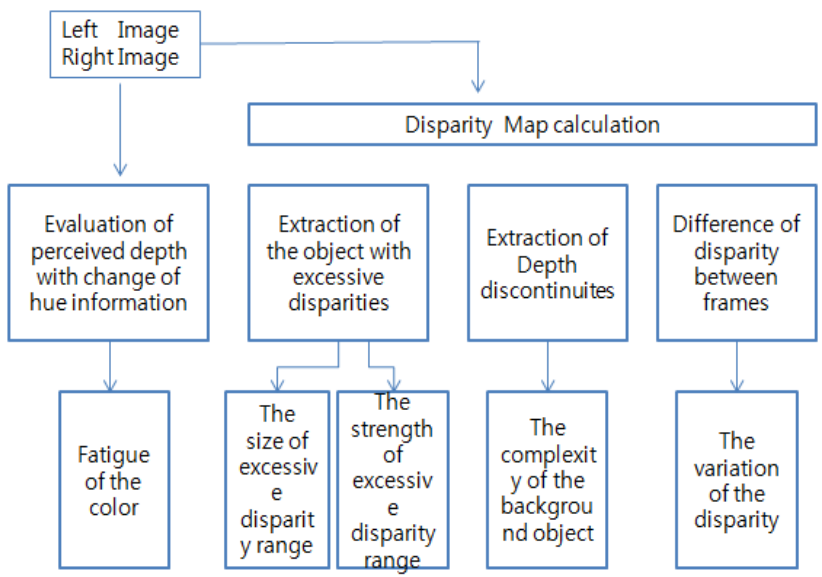

Fig. 1. The procedure of the visual fatigue evaluation.

\section{B. Extraction of the Object with Excessive Disparities}

We propose the method to extract objects with excessive disparities in 3D stereoscopic images using cost function considering the pixel intensities and the depth information, disparities.

The block diagram of the proposed method is as follows: In Fig. 2, the first the proposed method generates the disparity-map based on left image. The histogram is 
calculated from the disparity-map. The excessive disparity candidate regions are decided using the pre-defined threshold in the disparity-map. The extracted excessive disparity candidate regions are labeled to object regions and the other regions are labeled to background regions. The regions labeled as object are set as region that will be segmented in 3D stereoscopic left image. The proposed segmentation method considering the intensity and the depth information is applied to these regions in 3D stereoscopic left image. Finally to eliminate the protruding regions or the small regions, the morphological filter by means of the post processing is used.

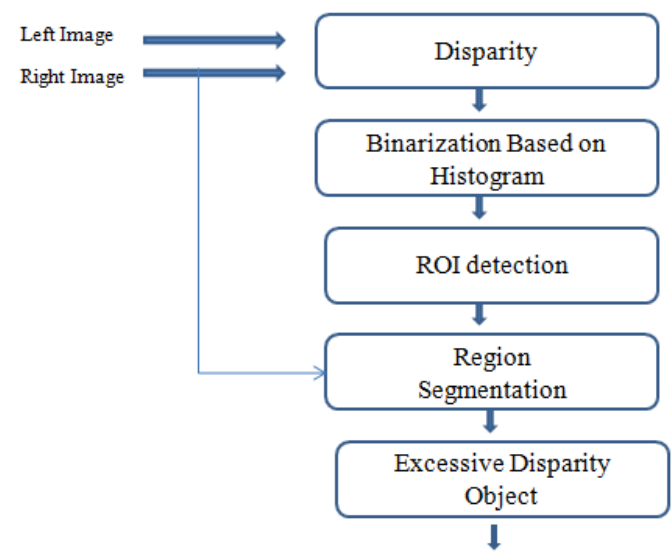

1.The size of the excessive disparity object 2.The strength of disparity of the excessive disparity object

Fig. 2. Block diagram of the proposed method.

\section{Extraction of the Depth Discontinuities}

The structure to extract the depth discontinuities is consist of four step, disparity map calculation, noise reduction, post processing, discontinuities extraction.

The proposed system is presented in Fig. 3. Disparity map is extracted from two images of the stereoscopy. Holes which occur in occluded regions should be filled before next process. Hole is filled with the interpolation of neighbor pixels. Next, Edge is detected from the pre-processed disparity map by DIP. Detected edges from the disparity map are depth discontinuities. However, edges which occur in the horizontal lines of the desk, ground are thick. So, in post processing, edges are made to be thin.

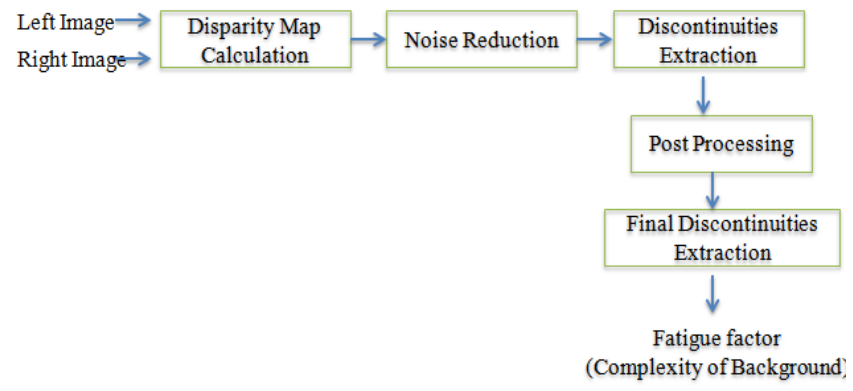

Fig. 3. The structure of the proposed system.

Disparity map calculation: Disparity map is extracted by the method proposed by Andres Geiger [13]. This method decrease stereo matching ambiguities using a prior distribution estimated from robust support points. Support points are reliable correspondences, which give valuable information to disambiguate remaining ambiguous disparities. 2D mesh via Delaunay triangulation is computed using a sparse set of support points. When creating 2D mesh, a prior is used to make the process efficient by restricting the search to plausible regions.

Noise Reduction: There are many holes in the disparity map due to occluded region. In the next step, edge detection from the disparity map by DIP, pixels would be the denominator of the equation to calculate the gradient of the depth. If the value of the pixel is a zero, this pixel is neglected in the extraction of the edge, that is, depth discontinuity because of the reason above mentioned reason. However, neglecting the zero pixel, the hole, would be the cause of the disconnected edge in edge detection by DIP. Therefore, the hole should be filled with non-zero value.

To fill these holes, we calculate the value of pixel in holes using the horizontally and vertically interpolating value of the pixel in the holes' boundary. Equation (2) shows the interpolation of the pixel in the hole with the nearest neighbor pixels.

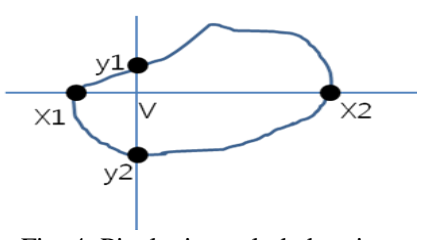

Fig. 4. Pixel $v$ in occluded region.

The pixel $v$ is a pixel in the hole, $x_{1}, x_{2}, y_{1}, y_{2}$ are pixels in the boundary which meets horizontally and vertically extended line. If $v$ is in the occluded region, the intensity of the $v$ is interpolated with the differently weighted $x_{1}, x_{2}, y_{1}, y_{2}$.

$$
\begin{gathered}
I(v)=\frac{\left(x_{1} w_{1}+x_{2} w_{2}\right)+\left(y_{1} w_{3}+y_{2} w_{4}\right)}{2} \\
w_{1}=\frac{\operatorname{dist}\left(x_{1}, v\right)}{\operatorname{dist}\left(x_{1}, x_{2}\right)} w_{2}=\frac{\operatorname{dist}\left(x_{2}, v\right)}{\operatorname{dist}\left(x_{1}, x_{2}\right)} \\
w_{3}=\frac{\operatorname{dist}\left(y_{1}, v\right)}{\operatorname{dist}\left(y_{1}, y_{2}\right)} w_{4}=\frac{\operatorname{dist}\left(y_{2}, v\right)}{\operatorname{dist}\left(y_{1}, y_{2}\right)}
\end{gathered}
$$

$\operatorname{dis}(x, y)$ is the distance of pixel $x$ and $y$.

In Eq.(2), $w_{1}, w_{2}, w_{3}, w_{4}$ is obtained with the ratio of the distance from pixel $v$ to neighbor pixels.

The extraction of the final depth discontinuities : DIP is the algorithm to extract edges from 2D image using the information of the intensity of pixels. The intensity of the 2D image is the brightness or materials in contrast with the depth or layers of the disparity map. Because of the similarity of the depth discontinuity in depth map and the edge of the image in the side of the using of the information of the gradation of the intensity, DIP could extract depth discontinuities from disparity map. DIP extract a pixel as an edge when the difference is lower than a threshold.

DIP is the algorithm to detect edges which are the regions involving abrupt changes of intensity, and valleys which are the regions composed of local intensity minima [14].

Valley is very important in vision. Various methods to detect valley like as Laplacian, Pearson's logical valley use the gradient values of the pixels in a local region. However Laplacian is too sensitive to noise and Pearson's logical 
valley cannot sometimes extract valleys which have somewhat small rates of change of intensity.

The entropy operator computes the entropy of intensity in a local region. This method depends on the local intensities, therefore, can extract the edges of dark regions very well. The disadvantage of this method is that it extract edges as thick lines and cannot respond in valleys very well.

The human viewer is more sensitive to the edges and valleys in dark regions than those in bright regions. Therefore, to perceive and analyze objects in a manner akin to the human visual system, one must extract sketch features subject to the local intensities. DIP satisfies the necessity for the perception and analysis of the human visual system as mentioned above. DIP is the in defined as (4).

$$
\begin{gathered}
D P(m, n)=\frac{I_{m}(m, n)}{\bar{I}(m, n)}-\frac{I(m, n)}{\bar{I}(m, n)} \\
D I P(m, n)=D P \frac{\bar{I}(m, n) \bar{I}(m, n)}{I_{m}(m, n) I(m, n)}
\end{gathered}
$$

$I(m, n)$ is the intensity of the pixel $(m, n), \bar{I}_{m}(m, n)$ is the sum of intensities and $I_{m}(m, n)$ is the maximum intensity in a window, In (3) and (3). Due to $I_{m}(m, n)$, the value of DP depends on local intensities and difference make DP respond well in valleys and edges. However, DP has a defect of extracting neighbor pixels as well as the valleys themselves.

To remove this problem, if the difference of $I_{m}(m, n)$ and $I(m, n)$ is greater than a given threshold obtained in (5) is applied. Each $\bar{I}_{m}(m, n) \quad I_{m}(m, n)$ are much the same in valleys and neighbors, while $I(m, n)$ is smaller in the valleys than in their neighbors. Thus, the values of DIP are so much larger in the valleys themselves that it extracts valleys thinly.

The result of the extraction is very various as to a threshold. As a threshold is so high, edge would be extracted too much. On the contrary to this, as a threshold is too low, edge would be extracted too less. Therefore, threshold is very important in the extraction of depth discontinuities. Usually, threshold is decided by user during the process. The interaction of the user during the process is improper to use depth discontinuity as a factor of the evaluation of the visual fatigue. We need a way to extract depth discontinuities to evaluate the visual fatigue in real time. To extract depth discontinuities in real time, a threshold should be decided without the interaction of the user efficiently.

Because the threshold should be decided to represent the characteristics of the disparity map, we learned threshold with the 10 image set in Middlebury dataset. We infer a threshold by the characteristics of the distribution of the depth map, that is, average and standard deviation. Table I shows average, standard deviation of the 10 image each and the result of the learned threshold.

After learning with average and standard deviation, we get an evaluation to decide the threshold and made a evaluation formula in (5) to decide a threshold to be representative the disparity map. The difference of the intensity of the disparity map means the depth of the difference, so, the value of threshold could be measured with mean and standard deviation of the depth [15].

$$
\operatorname{Threshold}(x)=-0.029 \times M(x)+0.014 \times S(x)+4.884
$$

where $M(x)$ is the mean and $S(x)$ is the standard deviation of the image $x$.

TABLE I: THE RESULT OF THE LEARNED THRESHOLD

\begin{tabular}{|c|c|c|c|c|}
\hline Name & $\begin{array}{c}\text { Average } \\
\text { (avg) }\end{array}$ & $\begin{array}{c}\text { Standard } \\
\text { Deviation } \\
\text { (std) }\end{array}$ & $\begin{array}{c}\text { Hand-tuned } \\
\text { threshold }\end{array}$ & $\begin{array}{c}\text { Learned } \\
\text { threshold }\end{array}$ \\
\hline aloe & 70.18 & 30.07 & 5.00 & 3.26 \\
\hline baby & 83.03 & 32.18 & 2.00 & 2.92 \\
\hline bowling & 115.53 & 59.46 & 2.00 & 2.35 \\
\hline flowerpots & 124.35 & 54.34 & 3.00 & 2.03 \\
\hline lampshade & 104.13 & 50.37 & 3.00 & 2.56 \\
\hline midd & 93.98 & 48.95 & 3.00 & 2.83 \\
\hline monopoly & 73.50 & 42.94 & 2.00 & 3.34 \\
\hline plastic & 135.33 & 40.35 & 1.00 & 1.52 \\
\hline rocks & 107.44 & 35.98 & 2.00 & 2.27 \\
\hline wood & 121.33 & 39.38 & 2.00 & 1.91 \\
\hline
\end{tabular}

Post process: The final process is a thinning after edge detected. If Object has a vertically different depth, there can be a thick edge during discontinuity extraction, so we have to thin a thick edge after edge detection. We employed zhang and seun [16] thinning algorithm. This thinning method obtain a new value from the previous iteration value. This method is called parallel method. This method is fast and simple to be implemented. So we would make a thick edge to be thin in depth Discontinuities.

\section{The Fatigue of the Color}

Kim et al. [17] included the parameters of color information into perceived depth model. They used color information as hue and lightness together. They proposed the perceived depth model as (6).

$$
D_{c}=\frac{v}{\frac{1}{S_{D} d}-1} \times f\left(\lambda_{1}, L_{1}, \lambda_{2}, L A_{2}\right)
$$

They concluded with (7) and showed the relationship between depth and wavelength, lightness.

$$
D_{c}=\frac{v}{\frac{1}{S_{D} d}-1} \times a \times\left(\frac{L_{1}}{0.0396 \lambda_{1}-0.0299 \lambda_{2}}-L_{2}\right)
$$

In this paper, we would analyze the perceived depth information in the form of offset rather than scale as Kim et al. did. Because they used the concept of color contrast, therefore the offset is proper than the scale.

In this paper, the perceived depth model is considered as the offset of depth information with the change of color contrast.

$$
D_{c}=\frac{v}{\frac{1}{S_{D} d}-1}+D_{\text {off }}
$$


We are interested in the parameter of $D_{\text {off }}$ The relationship between hue combination and $D_{\text {off }}$ is tested and analyzed in this paper. The test image set were generated as video with running time of 16 seconds showed in Fig. 5. We used two balls as the test object and set the color of two balls varying the color from $C 1$ to $C 13$. The left ball is called as $C_{\text {Left }}$ and right one as $C_{\text {Right }}$. We made all 78 set of test video with all possible color combinations using 13 colors from $C 1$ to $C 13$. The combination was performed as $\left(C_{\text {Left }}, C_{\text {Right }}\right)$ like $(C 1$, $C 2),(C 1, C 3), \ldots$ and $(C 12, C 13) . C_{\text {Left }}$ ball was put on left side of the scene and starts to move close to the eye at $T=0 \mathrm{sec}$. This ball arrives to the position most close to the eye at $T=8$ sec, and it moves the reverse route it already moved and finally to the initial position at $T=16 \mathrm{sec}$.

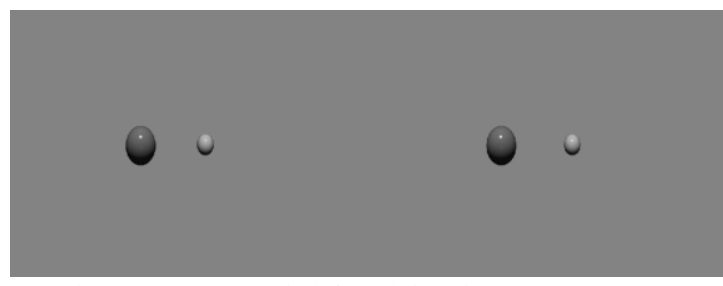

Fig. 5. Test stereopsis left and right image at $T=8 \mathrm{sec}$.

The test was performed as follows:

Step 1: Test video is displayed on the 3D-TV at $T=0$

Step 2: The video starts to play. $C_{\text {Left }}$ and $C_{\text {Right }}$ moves according to the route pre-defined.

Step 3: Observer find out the exact position of frame number that guarantees $C_{\mathrm{Left}}$ and $C_{\text {Right }}$ are on the same distance from the observer. We call this point as 'Forward match point'.

Step 4: Observer continues to watch the video and find out the next position of frame number when $C_{\text {Left }}$ and $C_{\text {Right }}$ meets again. We call this point as 'Backward match point'.

Step 5: Test above 4 steps for 78 video sets.

By the whole test, we can find out the exact forward and backward frame number for the matching. Then, this frame number can be converted into distance information because all 480 frames displayed corresponds the maximum moved distance of $C_{\text {Left }}$ and $C_{\text {Right }}$ each. Therefore, we can estimate the relationship between hue change and relative perceived depth offset as described above.

TABLE II: COLOR FATIGUE

\begin{tabular}{|l|l|l|l|l|l|}
\hline & Color $F$ & Color $B$ & $\lambda F$ & $\lambda B$ & $C$ (constrast) \\
\hline Case1 & Red & Green & 780 & 560 & 1.393 \\
\hline Case2 & Red & Blue & 780 & 440 & 1.773 \\
\hline Case3 & Green & Blue & 560 & 440 & 1.273 \\
\hline
\end{tabular}

We found if we decrease saturation values for some object in the scene, it looks like more farther than its real location through the test described above. This fact can be utilized in reducing the viewing fatigue for $3 \mathrm{D}$ stereoscopic images because reduce in saturation values to some extent causes the change of perceived depth information without any distortion in depth. Therefore, the viewing fatigue can be controlled in some extent without distortion of depth information.

The $C$ (contrast) increase if the color of the object is 'forward color', while decrease if the color of the object is 'backward color'. As to this rule. we can calculate the fatigue factor $C$ (contrast) in Table II for the image showed in Fig. 6. $\lambda F$ is the fatigue of the foreground object. $\lambda B$ is the fatigue of the background object. The fatigue of the color of the frame can be calculated by $\lambda F / \lambda B$. In this case the case 2 is more discomfort compared to others.

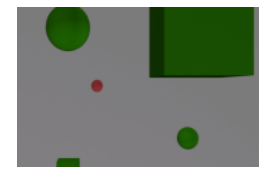

<case1>

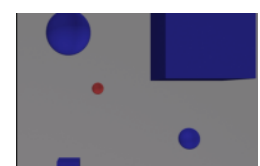

$<$ case $2>$

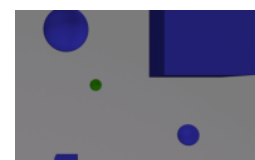

$<$ case $3>$
Fig. 6. Stereoscopic images that have different contrast between background object and moving object.

\section{EXPERIMENT}

\section{A. Experiment Environment}

The goal of the test is to verify the usability of the content independent visual fatigue factors used in fatigue evaluation model proposed this paper. The correlation of visual fatigue and visual factors, color, complexity of background object, excessive disparity, disparity variation, are tested on subject assessment.

The environment is composed of different level of degree with respect to visual factors. Color levels are prepared three different background object, movable object color pairs, (Red, Green), (Red, Blue, Green, Blue). The complexity of the background object is leveled three with the number of circle and square object. Level 1 of the complexity has two circles, one square, level 2 of the complexity has three circles, two squares, level 3 of the complexity has four circles and three squares. The level of size of excessive object is leveled with the size of radius of circle going forward in the content. The radius as to levels is $50 \mathrm{px}, 100 \mathrm{px}, 150 \mathrm{px}$ each. The variation of disparity is leveled with the speed of the circle going forward. Circle going forward 1px per frames in level 1 of variation of disparity, whereas, $2 \mathrm{px}$ in level 2, 3px in level 3 .

Fig. 7. shows the level 3 in color, complexity of the background, the size of object going forward. The number of all composition case made by each level of four visual factors is 81 .

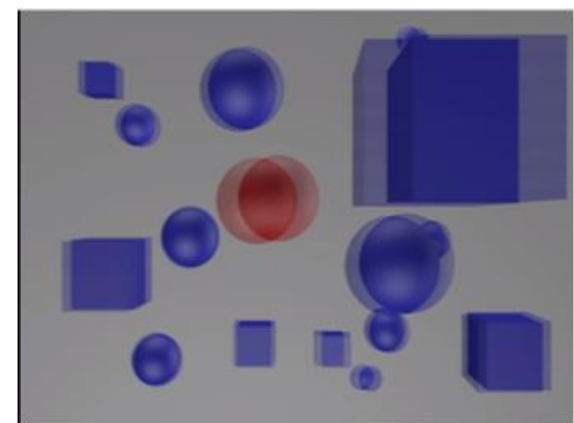

Fig. 7. The test stereoscopic video.

\section{B. Experiment Result}

The Score for the visual fatigue is obtained using three questionnaires, that is, tiredness of eyes, blurring of the eye, headache.

In Fig. 8 shows the score of the subjective assessment.

The complexity of the background, the speed of moving object which induce larger variation of disparity between frames are good indicators to evaluate the visual fatigue, 
whereas, the size of moving object which is proportion to the excessive disparity size seems to be low to impact on visual fatigue.

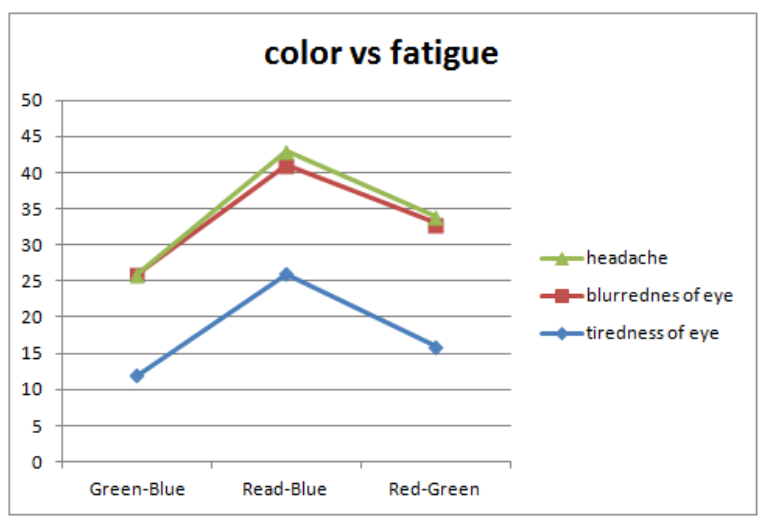

(a) the score of fatigue as to background and foreground color

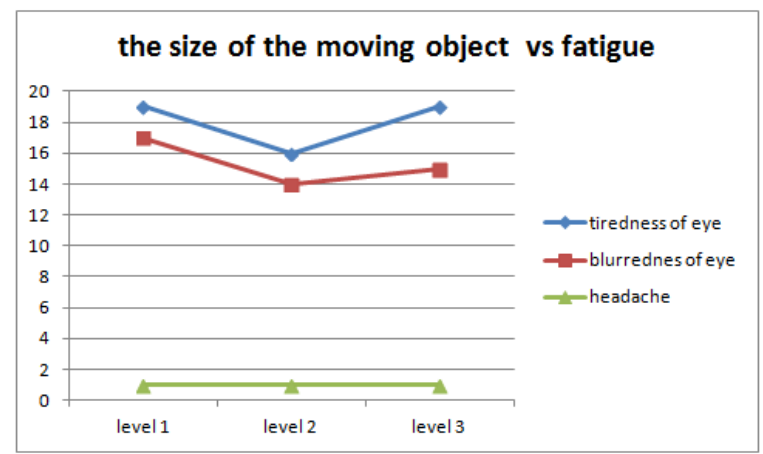

(b) the score of fatigue as to level of the size of the moving object

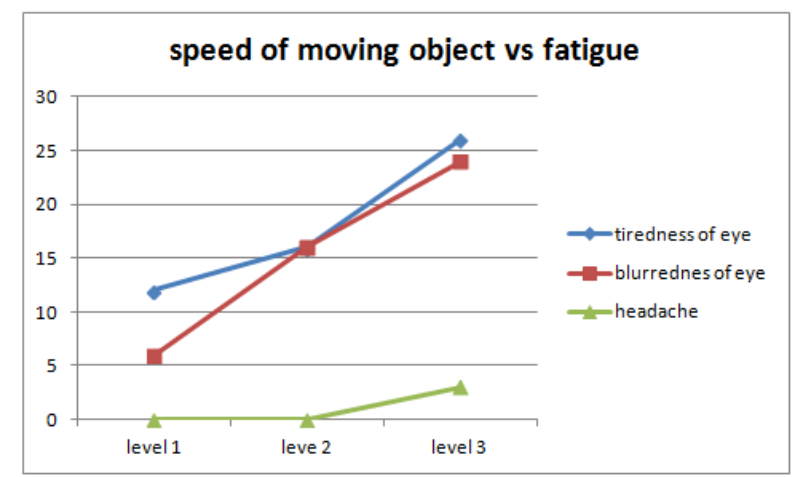

(c) the score of fatigue as to level of the speed moving object

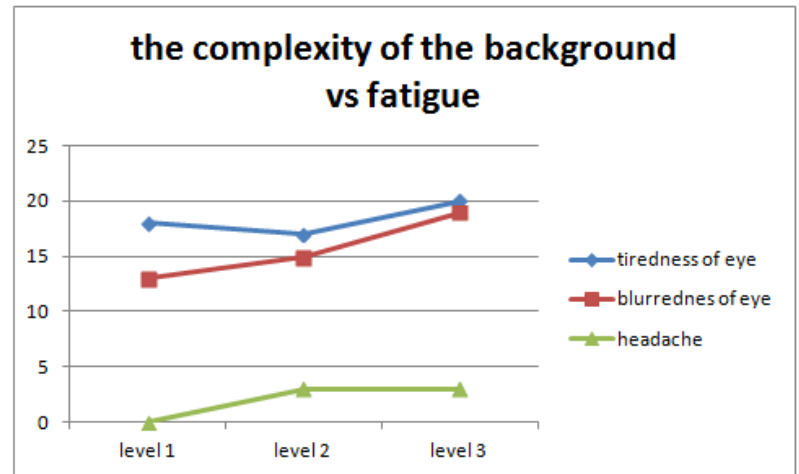

(d) the score of fatigue as to the level of the complexity of the background

Fig. 8. The result of the visual fatigue using subjective assessment.

\section{CONCLUSION}

There are not many researches to evaluate the visual fatigue of stereoscopic video objectively using the information of the video. In this research, we propose the content based factors and the model to evaluate the visual fatigue of the video using content based factors which can be extracted from the video automatically. We select five content factors of stereoscopic $3 \mathrm{D}$ video that are able to induce visual fatigue, the size and strength of the excessive disparity range, the complexity of the background object, the perceived depth of the hue, and the variation of the disparity between frames in pixels, as arguments of the evaluation model. In the experiment, the excessive disparity size seems to be low to impact on visual fatigue. There need to be more research to find other content factors that are high impact on the visual fatigue of the $3 \mathrm{D}$ stereoscopic video.

\section{ACKNOWLEDGMENT}

This research is partially supported by Ministry of Science, ICT and Future Planning(MSIP) and Institute for Information \&communications Technology Promotion(IITP) in the Information Communication Technology(ICT) and Culture Technology (CT) Research \&Development Program 2014.

\section{REFERENCES}

[1] K. Ukai and P. A. Howarth, "Visual fatigue caused by viewing stereoscopic motion images:Background, theories, and observations," Science Direct, Displays, vol. 29, pp. 106-116, 2008.

[2] M. T. M. Lambooij, W. A. IJsselstejin, and I. Heynderick, "Visual discomfort in stereoscopic displays: A review," SPIE-IS\&T, vol. 6490 , 2010.

[3] Y. Nojiro, H. Yamanoue, A. Hanazato, M. Emoto, and F. Okano, "Visual comfort/discomfort and visual fatigue caused by stereoscopic HDTV viewing," in Proc. SPIE-IS\&T Electronic Imaging, vol. 5291, 2004.

[4] S. Yano, M. Emoto, and T. Mitsuhashi, "Two factors in visual fatigue caused by stereoscopic HDTV images," Displays, vol. 25, pp. 141-150, 2004.

[5] M. Emoto, Y. Nojiri, and F. Okano, "Change in fusional vergence limit and its hysteresis after viewing stereoscopic TV," Displays, vol. 25, pp 67-76, 2004

[6] Y. Nojiri, H. Yamanoue, S. Ide, S. Yano, and F. Okano, "Parallax distribution and visual comfort on stereoscopic HDTV," IBC, 2006.

[7] S. H. Cho and H. B. Kang. (2011). An assessment of visual discomfort caused by motion-in-depth in stereoscopic 3D video. [Online]. Available: http://dx.doi.org/10.5244/C.26.65

[8] S. H. Kim, M. J. Kim, and J. W. Kang, "An analysis of the visual characteristic of directing for three dimensional films," Journal of the Korea Contents Association, vol. 11, no. 2, pp. 229-237, 2011.

[9] Y. J. Jung, S. Lee, H. Sohn, H. W. Park, and Y. M. Ro, "Visual comfort assessment metric based on salient object motion information in stereoscopic video," Journal of Electron Imaging, vol. 21, issue 1, Feb. 2012.

[10] R. S. Kennedy, N. E. Lane, K. S. Berbaum, and M. G. Lilienthal, "A simulation sickness questionnaire (SSQ): An enhanced method for qualifying simulator sickness," International Journal of Aviation Psychology, vol. 3, pp. 203-220, 1993.

[11] J. Kuze and K. Ukai, "Subjective evaluation of visual fatigue caused by motion images," Displays, vol. 29, pp. 159-166, 2008.

[12] H. Sohn, Y, J. Jung, and S. Lee, "Predicting visual discomfort using object size and disparity information in stereoscopic images," IEEE Transactions on Broadcasting, vol. 59, no. 1, 2013.

[13] A. Geiger, M. Roser, and R. Urtasun, "Efficient large-scale stereo matching," in Proc. Asian Conference on Computer Vision, Nov. 2010, vol. 6492, pp. 25-38

[14] Y. D. Chun, S. Y. Seo, and N. C. Kim, "Image retrieval using BDIP and BVLC moments," IEEE Transactions on Circuits and Systems for Video Technology, vol. 13, no. 9, Sept. 2003.

[15] G.-J. So, S.-H. Kim, and J.-Y. Kim, "The extraction of depth discontinuities using disparity map for human visual fatigue," International Journal of Computer Theory and Engineering, vol. 6 , no. 4, pp. 330-335, 2014. 
[16] T. Y. Zhang and C. Y. Suen, "A fast parallel algorithm for thinning digital patterns," Comm. ACM, vol. 27, pp. 236-239, 1984.

[17] J.-Y. Kim, S.-H. Kim, and G.-J. So, "Perceived depth modeling based on color information," International Journal of Computer Theory and Engineering, vol. 5, no. 4, pp. 716-720, 2013.

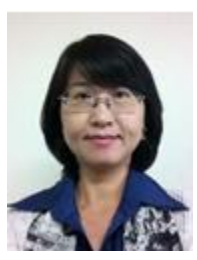

Gil-Ja So received the diploma in computer science from Dong-Eui University of Pusan, South Korea in 1994; M.S. and $\mathrm{PhD}$ degrees both in natural language processing of computer engineering, from the Pusan National university, South Korea in 1997 and 2012, respectively. From September 2001, she is with the Department of Computer Engineering of the University of Young-San at Yangsan, Kyungnam as a lecturer. Her doctoral dissertation work focused on the Korean grammar checker for parsing Korean documents and correcting context-sensitive words in documents. The topics she has worked on include virtual reality, game development and artificial intelligence of non-players of game, and 3D stereoscopic images. Her current research is on region-based intensity correction in 3D stereoscopic images.

She is a member of the KMMS (Korea Multimedia Society) and KIPS (Korea Information Processing Society).

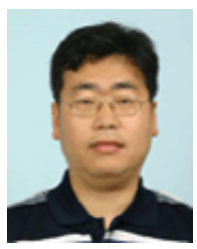

Sang-Hyun Kim received the diploma in electronics engineering from Kyung-Pook National University of Daegu, South Korea in 1991; M.S. and PhD degrees both in image processing of electronics engineering, from the same university, in 1993 and 1998, respectively.

He was with the Samsung Medison Co. Ltd, in Korea as a senior researcher from 1998 to 2001. From September 2001, he is with the Department of Computer Engineering of the University of Young-San at Yangsan, Kyungnam as an associate professor. His doctoral dissertation work focused on the development of fractal image coding method and transmission for image processing, image communication and computer vision problems. The topics he has worked on include virtual reality, ultrasound image processing, medical image processing, image feature extraction and classification, and 3D stereoscopic images. His current research is on region-based intensity correction in 3D stereoscopic images.

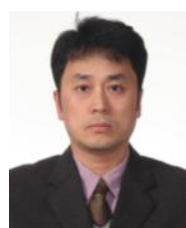

Jeong-Yeop Kim received the diploma in electronics engineering from Kyung-Pook National University of Daegu, South Korea in 1990; M.S. and PhD degrees both in image processing of electronics engineering, from the same university, in 1992 and 2000, respectively.

He was with the Samsung Advanced Institute of Technology in Korea as a senior research staff from 1994 to 2000. From March 2001 to February 2013, he was with the Department of Game Contents Science of the University of Young-San at Busan, as an associate professor. From March 2013, He is with the Department of Undeclared Major of the University of Young-San at Busan. His doctoral dissertation work focused on detecting and adjusting the white point from single color image. The topics he has worked on include color printing, error diffusion, color correction and 3D stereoscopic images. His current research is on correction of color data in 3D stereoscopic images. 